\title{
Numerical Cooking for Pasteurized Soft Boiled Eggs
}

\author{
Marjan Jenko \\ University of Ljubljana, Faculty of Mechanical Engineering, Slovenia
}

The industrial preparation of pasteurized soft boiled eggs requires meticulous planning of the thermal process. Requirements on keeping the yolk liquid and on extinction of potential salmonella allow little leeway in the creation of this process. The variation of the eggs' properties adds to the complexity. Thermal simulation of heat transfer within an egg is needed to get correlation data between transient temperature distribution and the egg's dimensional and material properties. A fast simulator of conductive transient heat transfer with a fixed grid of cells is developed for this purpose. The motivation for achieving the highest simulation speed was the potential integration of a simulation tool for simulation based predictions into an embedded control system. The simulated volume is a cylinder. The simulated object (the egg) is defined within the cylinder. Simulation results are analysed and used in the creation of the thermal process which results in certified pasteurized soft boiled eggs. The presented approach to the design of transient simulation can be used for applications ranging beyond the transient thermal simulation of foods. It can be adapted for any transient simulation where the local temporal intensity of changes depends on gradients and the properties of the matter.

Keywords: fixed grid simulation, conductive heat transfer simulation, transient thermal simulation, Fourier's law of heat flow, pasteurized soft boiled eggs, foods processing

Highlights

- $\quad$ The development of a faster than real-time transient thermal 2D simulation tool is presented in this paper.

- Faster than real-time simulation can be an integral part of a control system.

- The developed transient thermal simulation runs about 100 times faster than an equivalent commercial CFD simulation.

- The approach can be adapted for any transient simulation where the local temporal intensity of changes depends on gradients and on the properties of the matter.

- $\quad$ The test case is on sensitivity of temperature field dynamics to the dimensional and material properties of an egg.

- A unique patented thermal process for the production of pasteurized soft-boiled eggs is based on the results of the simulation.

\section{INTRODUCTION}

Numerical methods of solving differential equations that describe real world events are just about as old as the differential calculus itself. In such problems it is not necessary to aim at absolute mathematical exactness, because theoretical results that exceed in accuracy the tolerances of the best available observational instrumentation are not needed. Thus, differentials may be substituted for finite differences. This reduces practically insolvable problems in analysis to finite numerical calculations that can be done by computer. Yet, even so, partial differential equations can be quite challenging, both to processing units and memory space. The general objective is to ensure the convergence of algorithms within the limitations of computers. The Finite Elements Method (FEM) has been developed to this end. It is a method where one adjusts to the given problem the sizes of the finite elements, making them as large as possible in regions of space where the relevant functions are very smooth and as small as necessary where they are not for example, near sharp edges in boundary conditions. A disadvantage of the method of finite elements is the complexity of implementing it: not many engineers, who may need it, can write their own. Moreover, generalpurpose commercial packages are never optimally adapted to a particular problem. However, optimal adaptation is essential in the battle between the steadily decreasing limitations of computers and our steadily increasing objectives.

Faced with a practical problem of designing an apparatus with conceptually new functionality for an industrial client, this author was considering different options for the control of a thermal process that would produce pasteurized soft boiled eggs. One of the considered options was the development of a faster than real time transient thermal simulator, which could add to the process control. Meshing would be replaced with a predetermined fixed grid of cells and iterative procedures of the simulated case would need to be heavily optimized for speed. The whole task ought to be feasible to even moderately proficient programmers. Writing a simulation program for the problem in question (heating of an egg in water) confirmed the feasibility speculation.

The basic aim in developing the simulation was to identify the correlation between an egg's spatial distribution of temperatures while being heated and the egg's dimensional and material properties. This 
insight was needed firstly to decide on the viability of the new thermal process. Later the insight was essential in the design of the process and in the design of the control system.

Pasteurization is a delicate temperaturedependent process. It is different from sterilization where there are no active microorganisms left after the procedure. Pasteurization is aimed at a drastic decrease of the number of micro-organisms in foods the reduction being in the range of multiple orders of magnitude. The remaining concentration of pathogens is supposed to be limited at such a low level that causing a disease is unlikely if foods are stored at a low enough temperature for a short enough time. In the case of eggs, one should be most concerned about a potential contamination with salmonella.

Different approaches have been studied and different procedures have been developed to decontaminate potentially contaminated eggs. A combination of temperature, time and possible additional stress is needed to decrease the live salmonella concentration to a harmless level. Pasteurization and dry heat are combined in [1] to destroy the bacteria. Refs. [2] to [4] define combinations of temperatures and times for pasteurization. Ref. [5] is about inactivating bacteria on the shell's surface by using temperature treatment. Other sources of energy have been studied for bacteria inactivation: Microwave heating [6], radio frequency (RF) energy [7], combination of pulsed electric fields and heat [8] and ionizing radiation [9].

To validate pasteurization, a thermometer is to be inserted into the coldest spot of the food. This point has to be at or above lowest pasteurization temperature for a shortest pasteurization time or longer [10]. The best protection against salmonella is processing foods at high temperatures. This is the reason why industrial preparation of eggs for breakfast and supper buffets in hotels and restaurants offer hard boiled and scrambled eggs (high temperature processing), but not soft boiled eggs (low temperature processing).

\section{THEORY - PROTOTYPE PROBLEMS}

While the final objective of the simulation project is to develop a fast interactive and potentially embedded simulator applicable to many practical problems, the development work is currently restricted to the conductive heating of an object. The specific model used is that of a cold egg being put in a pot of hot water. The simulator is to yield the temperature as the function of time for every point inside the egg - taking into account the different thermal properties of the shell, of the white, of the yolk, and of the air pocket. At a subsequent stage of development, one could take into account the modifications of these properties during cooking, but it is important to realize that these modifications should not affect the underlying simulation engine.

In general, the egg problem is three-dimensional, but due to rotational symmetry with respect to the egg's axis, it reduces to a two-dimensional problem. For spherical eggs, it reduces to a one-dimensional problem. Thus, a problem's dimensionality is not necessarily the same as the dimensionality of the object in question. Thus, while all objects are threedimensional, some may be modelled as if they were of lower dimension.

\section{METHODS - DEVELOPMENT OF A FIXED GRID SIMULATOR FOR CONDUCTIVE HEAT TRANSFER}

One assumes that the initial temperature of the egg is uniform and that the water bath is at a constant temperature.

The two dimensions are radial and longitudinal. The latter will be called axial.

For numerical simulation, the egg is centred in a slightly larger cylinder subdivided into small cells. We refer to this cylinder as the frame.

Each cell is labelled by two integers: The axial coordinate $i$, and the radial coordinate $j$. The corresponding coordinates in millimetres are $x$ and $y$.

In the graphical representation, which shows an egg in longitudinal cross section, the pair of integers $(i, j)$ represents a pixel.

This implies that the centre-to-centre distance of all neighbouring cells is the same along both coordinates. This universal distance is denoted by $d$ and measured in millimetres. A value of about $0.15 \mathrm{~mm}$ (the thickness of a sheet of paper) seems intuitively small enough, and also works well.

\section{Axial Decomposition:}

The 'slicing' of the cylindrical frame along the axial direction is shown in Fig. 1.

The left-most slice is $i=0$. The rightmost is $i=\mathrm{N}$.

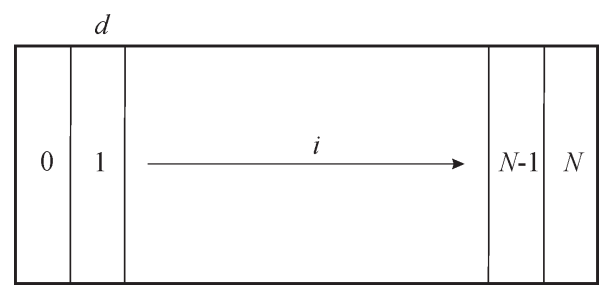

Fig. 1. Axial decomposition of the cylindrical frame 


\section{Radial Decomposition:}

Fig. 2 shows the radial subdivision of the cylinder into rings. The central cells $(j=0)$ are solid cylinders of radius $d$ and height $d$. All other cells are rings of thickness $d$. The last ring is $j=\mathrm{M}$.

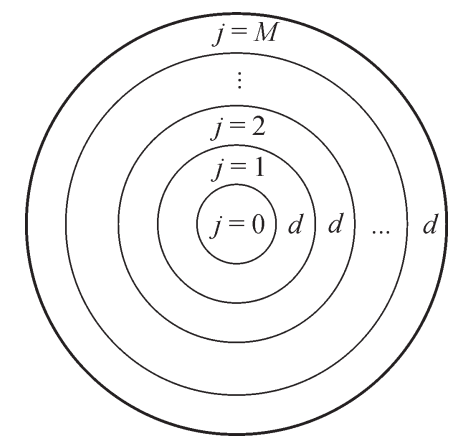

Fig. 2. Radial decomposition of the cylindrical frame

\section{The geometric functions:}

In Fig. 3, a typical cell $(j>0)$ is shown in axial view as a ring of width $d$ (and height $d$ ). The parameters specifying the cell are defined in Fig. 3.

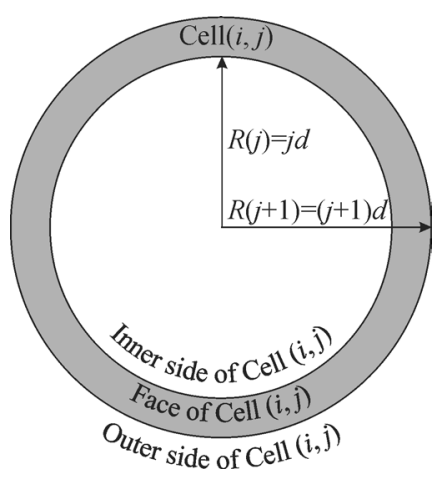

Fig. 3. A typical cell in the axial view

The cells are bounded by sides and by faces.

Radii: $R(j)$ is the radius of the inner bounding side of cell $(i, j)$. Thus, $R(0)=0$ because the central cells, cell $(i, 0)$, are solid cylinders. In general,

$$
R(j)=j d .
$$

Sides: $S(j)$ is the area of the inner side of cell $(i, j)$. It is the perimeter $2 \pi R(j)$ times the height $d$. Thus

$$
S(j)=2 \pi R(j) d .
$$

Hence, by Eq. (1):

$$
\begin{gathered}
\text { inner side: } S(j)=2 \pi d^{2} j, \\
\text { outer side: } S(j)=2 \pi d^{2}(j+1) .
\end{gathered}
$$

$$
F(j)=\pi\left((R(j+1))^{2}-(R(j))^{2}\right) .
$$

Hence, by Eq. (1),

$$
F(j)=\pi d^{2}(2 j+1) \text {. }
$$

Volumes: $V(j)$ is the volume of cell $(i, j)$. It is the area of face times the height $d$ :

$$
V(j)=F(j) d .
$$

Thus, by Eq. (6),

$$
V(j)=\pi d^{3}(2 j+1)
$$

\section{Distances between cells:}

As shown in Fig. 1, the axial distance between cell $(i, j)$ and cell $(i+1, j)$ is $d$ for all $i$ and all $j$.

As shown in Fig. 2, the radial distance between cell $(i, j)$ and cell $(i+1, j)$ is $d$ for all $i$ and all $j>0$. For $j=0$, it is $3 d / 2$. Thus:

$$
\operatorname{dist}(0,1)=\frac{3}{2} d, \quad \operatorname{dist}(1,2)=d \quad \text { etc. }
$$

\section{Fourier's law of heat flow and the energy as a function of temperature:}

We are to apply the Fourier's law of heat flow:

$$
\frac{d Q}{d t}=G A \frac{d T}{d x},
$$

and the heat energy as a function of temperature:

$$
Q=C V T,
$$

to our discrete problem.

\section{Discretization:}

The differentials in Eq. (9) are to be replaced by finite differences:

$$
\Delta Q=G A \frac{\Delta T}{\Delta x} \Delta t,
$$

which must be sufficiently small for the simulation algorithm to converge.

In Eq. (11), $\Delta x$ represents the axial as well as radial distance between the centres of neighbouring cells:

$$
\Delta x=d
$$

The elementary time interval is denoted by $\tau$,

$$
\Delta t=\tau .
$$

Faces: $F(j)$ is the area of each of the two faces of cell $(i, j)$. It is the difference of two disk areas: 


\section{Implementation:}

Let temperature and heat contents be stored in arrays $T(N, M)$ and $Q(N, M)$. The fundamental functions $T(x, r)$ and $Q(x, r)$ are thus represented by the array elements $T(i, j)$ and $Q(i, j)$.

In Eq. (10), the volumetric heat capacitance $C(i, j)$, the volume $V(i, j)$, and the temperature $T(i, j)$, all belong to the same cell $(i, j)$.

In Eq. (11), different cells are involved: as shown in Fig. 4, $A$ is the area of the bounding surface, $G$ is the average conductivity of two cells, $\Delta T$ is the temperature difference between the cells, and $\Delta Q$ is the heat flow through $A$ in the time interval $\tau$. Specifically:

the average axial conductivity $G_{a}$ of the cell $(i, j)$ is:

$$
G_{a}(i, j)=2 \frac{G(i, j) G(i+1, j)}{G(i, j)+G(i+1, j)},
$$

and the average radial conductivity $G_{r}$ of the cell $(i, j)$ is

$$
G_{r}(i, j)=2 \frac{G(i, j) G(i, j+1)}{G(i, j)+G(i, j+1)} .
$$

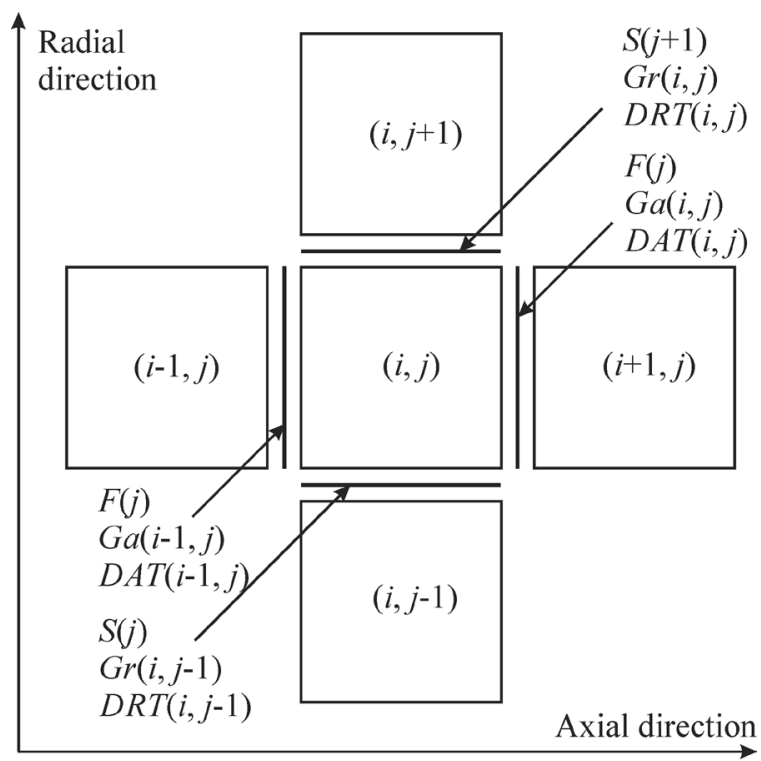

Legende: $S$ - side area, $F$ - face area,

$G r$ - average radial conductivity,

$G a$ - average axial conductivity,

$D R T$ - difference radial temperature,

$D A T$ - difference axial temperature.

Fig. 4. The cell (i,j) and adjacent cells

To see why, let us think in terms of thermal resistance from centre-to-centre.
The temperature differences DAT (difference axial temperature) and $D R T$ (difference radial temperature) at any instant in time are defined as:

$$
\begin{aligned}
& \operatorname{DAT}(i, j)=T(i+1, j)-T(i, j), \\
& \operatorname{DRT}(i, j)=T(i, j+1)-T(i, j) .
\end{aligned}
$$

Fig. 4 and Eq. (9) yield the heat flowing in the time interval $\tau$ into the cell $(i, j)$ from its four neighbouring cells:

$$
\begin{aligned}
D Q(i, j) & =D_{1} Q(i, j)+D_{2} Q(i, j)+ \\
& +D_{3} Q(i, j)+D_{4} Q(i, j),
\end{aligned}
$$

where from top:

$$
D_{1} Q(i, j)=+\frac{\tau}{d} S(j+1) G_{r}(i, j) \operatorname{DRT}(i, j),
$$

from bottom:

$$
D_{2} Q(i, j)=-\frac{\tau}{d} S(j) G_{r}(i, j-1) D R T(i, j-1),
$$

from right:

$$
D_{3} Q(i, j)=+\frac{\tau}{d} F(j) G_{a}(i, j) D A T(i, j),
$$

from left:

$$
D_{4} Q(i, j)=-\frac{\tau}{d} F(j) G_{a}(i-1, j) D A T(i-1, j) .
$$

\section{The T- $Q$ relation:}

By Eq. (10), the heat difference $D Q(i, j)$ produces a temperature difference $D T(i, j)$ in cell $(i, j)$ :

$$
T(i, j)=\frac{D Q(i, j)}{V(j) C(i, j)} .
$$

By components, from top:

$$
D_{1} T(i, j)=+\frac{\tau}{d} \frac{S(j+1) G_{r}(i, j)}{V(j) C(i, j)} D R T(i, j),
$$

from bottom:

$$
D_{2} T(i, j)=-\frac{\tau}{d} \frac{S(j) G_{r}(i, j-1)}{V(j) C(i, j)} D R T(i, j-1),
$$

from right:

$$
D_{3} T(i, j)=+\frac{\tau}{d} \frac{F(j) G_{a}(i, j)}{V(j) C(i, j)} D A T(i, j),
$$


from left:

$$
D_{4} T(i, j)=-\frac{\tau}{d} \frac{F(j) G_{a}(i-1, j)}{V(j) C(i, j)} D A T(i-1, j)
$$

We next introduce the Eqs. (3), (4) (6) and (8) for $S(j), F(j)$ and $V(j)$. After all cancellations, one obtains from top:

$$
D_{1} T(i, j)=+\tau \frac{2(j+1) G_{r}(i, j)}{d^{2}(2 j+1) C(i, j)} D R T(i, j),
$$

from bottom:

$$
D_{2} T(i, j)=-\tau \frac{2 j G_{r}(i, j-1)}{d^{2}(2 j+1) C(i, j)} D R T(i, j-1)
$$

from right:

$$
D_{3} T(i, j)=+\tau \frac{G_{a}(i, j)}{d^{2} C(i, j)} D A T(i, j)
$$

from left:

$$
D_{4} T(i, j)=-\tau \frac{G_{a}(i-1, j)}{d^{2} C(i, j)} D A T(i-1, j)
$$

Thus, at the cell $(i, j)$, the temporal temperature difference over the time interval $\tau$ is given in terms of the spatial temperature differences over the distance $d$ to the four closest neighbours. Introducing the notations:

$$
\begin{gathered}
K_{1}(i, j)=\frac{\tau}{d^{2}} \frac{2 j+2}{2 j+1} \frac{G_{r}(i, j)}{C(i, j)}, \\
K_{2}(i, j)=\frac{\tau}{d^{2}} \frac{2 j}{2 j+1} \frac{G_{r}(i, j-1)}{C(i, j)}, \\
K_{3}(i, j)=\frac{\tau}{d^{2}} \frac{G_{a}(i, j)}{C(i, j)}, \\
K_{4}(i, j)=\frac{\tau}{d^{2}} \frac{G_{a}(i-1, j)}{C(i, j)},
\end{gathered}
$$

we have

$$
\begin{aligned}
& D T(i, j)= \\
& =K_{1}(i, j) D R T(i, j)-K_{2}(i, j) D R T(i, j-1)+ \\
& +K_{3}(i, j) D A T(i, j)-K_{4}(i, j) D A T(i-1, j),
\end{aligned}
$$

$$
T(i, j) @(t+\tau)=T(i, j) @ t+D T(i, j) @ t .
$$

Since $\tau / d^{2}$ in Eqs. (32) to (35) can be treated as a given constant while the matrices $K_{1}(i, j)$ to $K_{4}(i, j)$ can be computed only once during simulation setup, the theoretical part of the problem is solved.

\section{SIMULATION}

\subsection{The Simulator Itself}

The simulator is coded in the environment of the $\mathrm{C}++$ Builder RAD tool. The display of its user interface is accessible at the http://www2.arnes.si/ mjenko9/ SimT/GUI.jpg. It is designed from the Visual Component Library objects. The code itself consists of close to $2000 \mathrm{C}$ lines. The majority of the code is about drawing results and facilitating user input. The actual micro stepping algorithm consists of less than three hundred ANSI C lines of code. 5 seconds of real time is simulated in 1 second of simulation time on a platform with the Intel i3 processor.

Thermal data that are used in the simulation are in Table 1 . Thermal conductivity and heat capacity data are derived from [11] to [14].

Table 1. Thermal parameters used in the simulation

\begin{tabular}{lcc}
\hline & $\begin{array}{c}\text { Thermal conductivity } \\
\mathrm{G}[\mathrm{W} /(\mathrm{mm} \mathrm{K})]\end{array}$ & $\begin{array}{c}\text { Heat capacity } \\
\mathrm{C}[\mathrm{J} /(\mathrm{K} \mathrm{mm})]\end{array}$ \\
\hline Water bath & 0.00060 & 0.00420 \\
\hline Shell & 0.00050 & 0.00200 \\
\hline Egg white & 0.00065 & 0.00120 \\
\hline Yolk & 0.00040 & 0.00300 \\
\hline Air pocket & 0.00010 & 0.00001 \\
\hline
\end{tabular}

\subsection{Simulated Cases}

Table 2 shows 14 simulation scenarios. Simulation results are in Fig. 5. The purpose of these simulations is to learn the influence of the egg's properties on the heat-up time of the coldest spot within the egg. The initial temperature of the egg is $5{ }^{\circ} \mathrm{C}$. The water bath is at $90^{\circ} \mathrm{C}$. In Fig. 5, a cross-section of the simulated egg is displayed at each of the 14 simulations. The line in the middle of the egg's cross section defines the position of the temperature profile, which is shown below the egg. The temperature profile is drawn each finished thirty seconds interval of the heat-up time. The time needed for the coldest spot within the egg to reach $60^{\circ} \mathrm{C}$, is marked and annotated. 


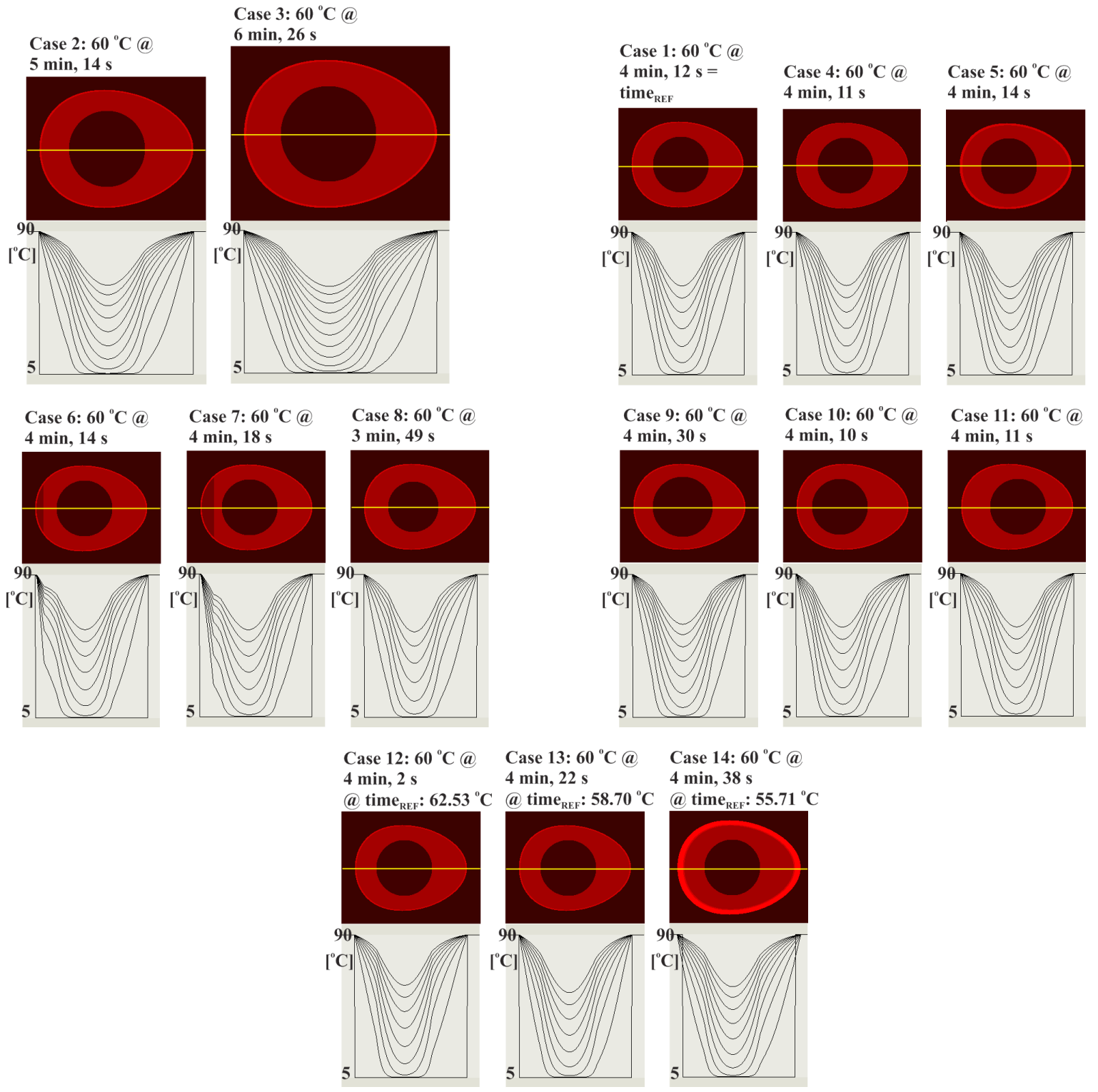

Fig. 5. Simulation results

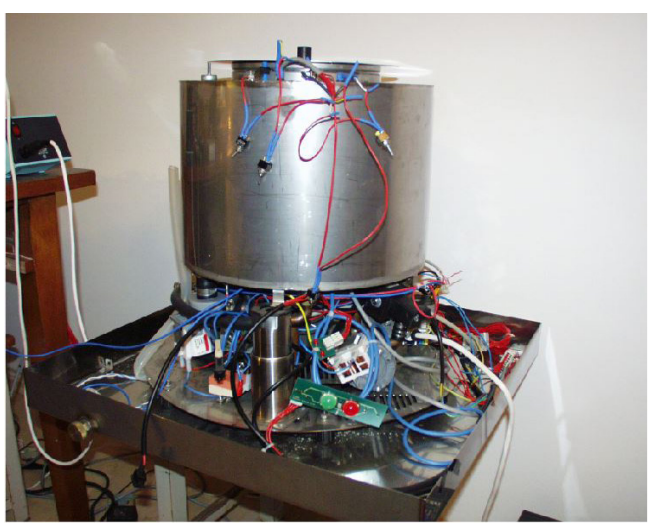

Fig. 6. Mechanical hardware of the laboratory system 
Table 2. Simulated cases of egg's variability

\begin{tabular}{cc}
\hline Case No. & egg's variant \\
\hline 1 & reference sized \\
\hline 2 & larger 1 \\
\hline 3 & larger 2 \\
\hline 4 & shell thickness $50 \%$ \\
\hline 5 & shell thickness $200 \%$ \\
\hline 6 & air pocket $5 \%$ egg's volume \\
\hline 7 & air pocket $10 \%$ egg's volume \\
\hline 8 & yolk volume $90 \%$ \\
\hline 9 & yolk volume $110 \%$ \\
\hline 10 & yolk to left $10 \%$ \\
\hline 11 & yolk to right $10 \%$ \\
\hline 12 & white G $110 \%$, white C $90 \%$ \\
\hline 13 & white G $90 \%$, white C $110 \%$ \\
\hline 14 & water cooled by egg's surface \\
\hline
\end{tabular}

The simulated cases 1 to 3 , size of an egg: influence of the egg's size on the heat up time is high. For a spherical object the surface area increases proportionally to the square of the radius and the volume increases proportionally to the cube of the radius. It is similar in the case of an egg. The volume takes up the heat energy, which first has to cross the surface. It is hence not surprising that heat up times of larger eggs are longer.

Cases 4 and 5, shell thickness halved and doubled: influence of shell thickness on the heat up time is not significant $(-0.4 \%,+0.8 \%$, compared with the reference time in the Case 1). The shell and other components of the egg have thermal properties of the same magnitude, but the shell is thin, compared to other geometries of the egg. Temperature distributions within an egg in simulated cases 4 and 5 are mostly similar. Halving and doubling the shell thickness has no significant influence either on the heat up time, or on the temperature distribution within an egg.

Cases 6 and 7, formation of an air pocket in an older egg: heat up times are increased by $0.8 \%$ and $2.4 \%$, which is not significant. The air pocket does influence the temperature distribution within the egg. The air pocket does not influence the location of the coldest spot, which is at the centre of the yolk.

Cases 8 and 9, influence of the yolk's size on the heat up time: a case with a smaller yolk decreases the heat-up time by $9 \%$, a case with a bigger yolk increases the heat up time by $7 \%$. Compared to the egg white, the yolk has lower thermal conductivity and higher thermal capacity which is the primary reason for these results.

Cases 10 and 11, influence of yolk's position on the heat-up time: heat up times do not significantly differ at different yolk positions. The temperature distribution is significantly correlated to the position of the yolk, but the coldest spot remains in the centre of the yolk.

Cases 12 and 13, thermal properties of the egg's white: heat up time is within $-4 \%,+10 \%$. The temperature at the yolk's centre (coldest spot) after the reference heat up time is $1.30{ }^{\circ} \mathrm{C}$ and $+2.53{ }^{\circ} \mathrm{C}$ off the targeted $60^{\circ} \mathrm{C}$.

Case 14, taking into account water cooling at the egg's surface: the heat-up time is increased by $10 \%$, which is significant.

\section{EXPERIMENTAL WORK}

\subsection{Design of Control System}

The project of making pasteurized soft boiled eggs involves biotechnology, modelling of heat transfer, and design of a mechatronic product within domains of software, electronics and hardware. A unique thermal process had to be developed and implemented.

The corresponding laboratory system was built with the ambition of being as flexible as possible when it comes to experimentation and to be reusable in the design of the apparatus itself. The mechanical hardware of the laboratory system is in Fig. 6.

The hardware consists of two containers filled with water, water cooler, pumps and valves. The system was built with the aim of heating and cooling up to 30 eggs that are immersed in temperature regulated circulating water. The laboratory system has no embedded control system but it is equipped with sensors and actuators. The custom electronics was designed later in the project.

The simultaneous design of the thermal process and of the governing software, without available target embedded electronics could be challenging. We decided to use a PC for the development platform. A data acquisition (DAQ) board was used as an interface between the PC and the sensors and actuators of the experimental system. Temperatures were measured with a temperature meter that is connected to the PC via the serial interface. The project is structured in interrelated software components in Fig. 7. ANSI C was used for the coding, with the aim of portability to the target system, as it became available. MS Windows, which is not a real-time OS, was running on the PC. Since thermal processes are slow (compared to computers) and software process priorities can be set, the Windows OS fits the purpose of this process control. The DAQ board is addressed exclusively via its' ANSI C application programming interface (API). 
Interrelated software components of the developed apparatus are in Fig. 8. About $40 \%$ of the process development code was ported to the target system with merely small modifications.

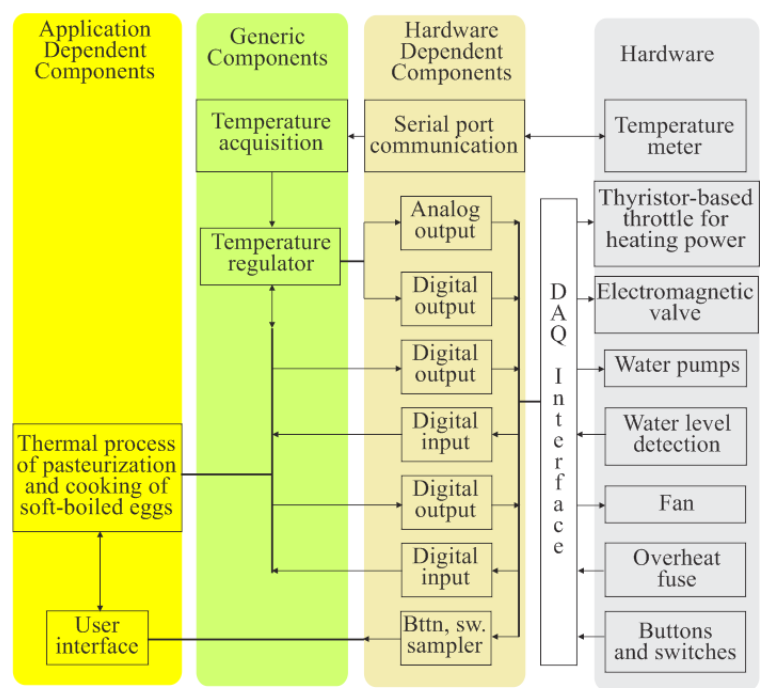

Fig. 7. Interrelated software components of the laboratory system

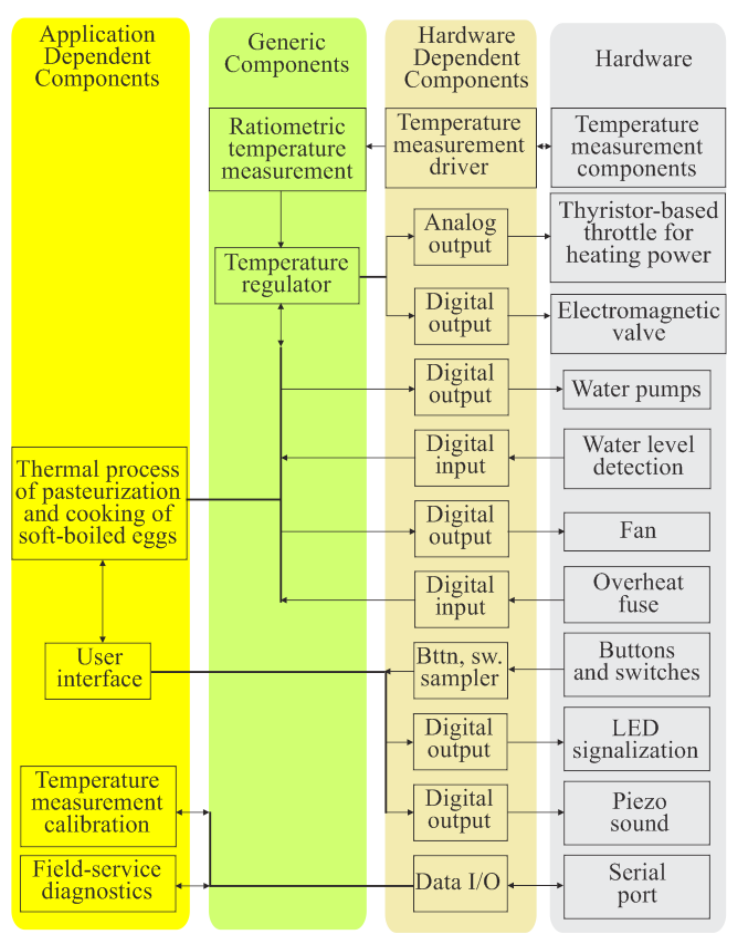

Fig. 8. Interrelated software components of the apparatus

\subsection{Matching of Simulation and Cooking}

The implemented temperature profile for the circulating water in the basin with eggs and measured temperatures in the centres of eggs are in Fig. 9. The designed temperature profile of the circulating water starts at a low enough temperature that the immersed eggs do not break due to thermal stress. The heating power is limited by the regulations imposed on electrical equipment $\left(I_{\mathrm{MAX}}=16 \mathrm{~A}\right.$, single phase, 3700 $\mathrm{W}$ with a $230 \mathrm{~V}$ grid). Water temperature is limited to $90^{\circ} \mathrm{C}$. After selected times (for different egg sizes) the water temperature drops to $60{ }^{\circ} \mathrm{C}$. At the same time, the coldest spot within an egg reaches $60^{\circ} \mathrm{C}$, which is the pasteurization temperature. 10 minutes later (not shown in Fig. 10) the temperature of the water bath drops to $57^{\circ} \mathrm{C}$, which is low enough for an egg to keep its' organoleptic properties and to stay warm till use.

The developed thermal process needs to be adjusted at the start of each run for the egg size (standard Euro size small (weight $\leq 53 \mathrm{~g}$ ), medium $(53 \mathrm{~g}<$ weight $\leq 63 \mathrm{~g})$, large $(63 \mathrm{~g}<$ weight $\leq 73 \mathrm{~g})$, very large (73 $g<$ weight), - all eggs in the basket are of the same declared size). The developed thermal process is regulated within $\pm 0.3{ }^{\circ} \mathrm{C}$ (temperature of the circulating water bath). This prevents variations in eggs' properties to have noticeable effects on the quality of the pasteurized soft boiled eggs. Fortunately, the thermal process does not have to be adjusted for the other egg parameters then size. Daily use of the apparatus in the field confirms the findings.

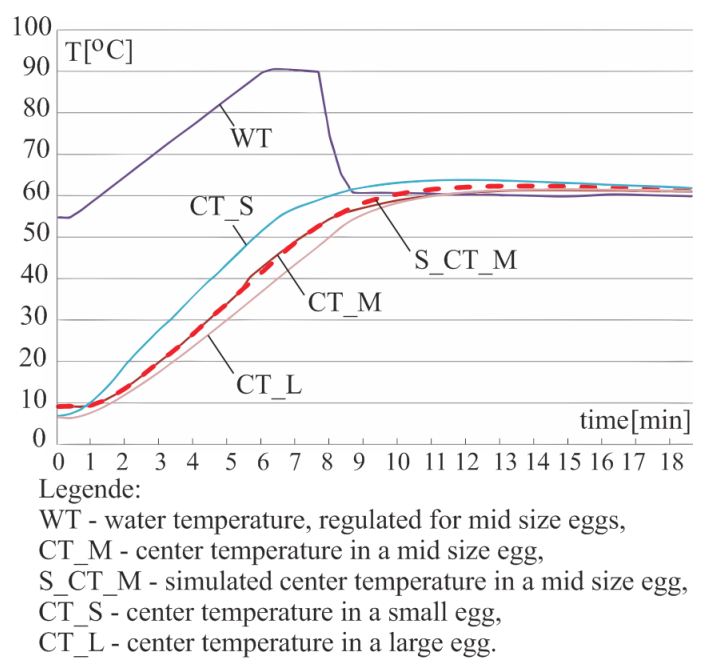

Fig. 9. Measured and simulated temperature profiles

\section{DISCUSSION}

We learned from the results of the thermal simulations that the heat-up time is significantly related to egg size, yolk size and water being cooled by the egg's surface. 
The heat-up time is far less related to the position of yolk, shell thickness, the formation of an air pocket and temperature induced variations of the egg white thermal properties. These relationships do not need attention in the production of pasteurized soft boiled eggs.

Heat is transferred by radiation, convection and conduction. Radiative heat transfer is not relevant when heating up an egg in a bucket of hot water. It is a different situation with convective heat transfer. Egg white is a thick liquid - as such it can contribute to heat transfer by convection. Nevertheless, we did not include this type of heat transfer in the simulation. Our reasoning for the exclusion of convective heat transfer from simulation is based on the results of previous experiments related to the thermal treatment of eggs. We had been experimenting with heatingup and cooking eggs of different sizes and ages and origins being stockpiled in a bucket made from a metal mesh. The bucket with the eggs was submersed into a reservoir with circulating hot water with a precisely regulated temperature. We did observe a strong correlation between egg size and invasively measured temperature in the centre of the egg. Measurements did have their own noise since the temperature sensor had to be installed in the centre of the egg. We did not observe significant correlation between the position of an egg in a bucket (laid on a side, on a sharp, on a soft tip) and by organoleptic properties of the produced soft boiled egg. This justifies the exclusion of convective heat transfer in the study.

The relevant similar literature on thermal simulation of eggs is provided in references [11] and [13] to [16].

The results of the CFD thermal simulation of an egg are compared to the thermal measurements in [13]. However, the test eggs are first hollowed out and then refilled with agar that has uniform thermal properties. The simulation correlates well with the measurements.

Ref. [14] presents a CFD thermal simulation of an egg, and studies the influence of the egg's rotation to the rate of the egg heating in water, with the aim of shortening the pasteurization process. Experimental work, i.e., temperature measurements are performed on eggs without yolk.

Ref. [15] is about another CFD thermal simulation and studies heat transfer from the egg's interior to the environment. This study is relevant to the embryotic development within an egg. Experimental work is performed with the help of an instrumented egg with built-in electrical resistors for heat generation.

Ref. [16] presents a CFD thermal simulation, which studies the combined conductive and convective heat transfer in model eggs that are filled with a sodium carboxy-methyl cellulose suspension with well-known uniform thermal properties.

Ref. [11] reports simulation and measurements of pasteurization process for liquid yolk, which is obtained from broken eggs.

Using the aforementioned published research, experimental verification of simulation results is not straightforward. Installing a grid of temperature sensors in an egg, without significantly changing its thermal properties, has not yet been achieved.

\section{On the simulator itself:}

Fourier heat equation dates from 1822. For the last thirty years the FEM and to the less extent the Finite Difference Method (FDM) have been utilized for steady state and transient modeling of heat transfer. In the case of both methods one firstly generates a grid with nodes. Secondly, the node temperatures are calculated for each time $t$ of the analysis. The whole simulated structure is analyzed as a unity, which implies that a system of equations is solved for each time $t$ of the transient analysis. To implement algorithms that solve a relatively large system of equations one needs a substantial amount of computer memory and processing power.

In the presented simulation there is no system of $n$ linearly independent equations to calculate $n$ variables at each time $t$. The objective is to have a faster than real-time transient thermal simulation that runs on a moderate computer platform - a lap top or an embedded system. The final motivation for this objective is the potential integration of a simulation tool for simulationbased predictions into an embedded control system of a modern high-tech mechatronic apparatus. Speed is always needed in interactive simulations.

The performance of embedded systems and personal computers is improving, but there will always be a certain lag between these systems and high end workstations. When it comes to the design of software that is to run on relatively modest platforms for real time applications (embedded systems), a) one needs to be careful about memory use, b) the frequently iterated segments of code need to be optimized for speed, and c) code has to be designed from the beginning with the aim of portability between different platforms - the code is developed on a capable workstation and then it runs in an embedded system. It is a de-facto standard to write code in ANSI C (compilation or crosscompilation for different platforms). Pre-compiled libraries need to be available for all platforms of the project. 
We did a test of simulation speed for an area of $50 \mathrm{~mm} \times 20 \mathrm{~mm}$ (size of the egg simulation) with the presented simulator (PS) and asked for evaluation of speed and memory using Dassault's ABAQUS for the same case. The ABAQUS speed and the requirement on memory are copied from the received file. Results are presented in Table 3 .

Table 3. PS and ABAQUS, speed and resources

\begin{tabular}{lcccc}
\hline & Case 1 & Case 2 & Case 3 & Case 4 \\
\hline memory [MB] & 10 & 3 & 28 & 80 \\
\hline $1 \mathrm{~s}$ of simulation & $5.00 \mathrm{~s}$ of & $2.10 \mathrm{~s}$ of & $0.05 \mathrm{~s}$ of & $0.003 \mathrm{~s}$ of \\
time at $\Delta t=15 \mathrm{~ms}$ & real time & real time & real time & real time \\
\hline
\end{tabular}

Legende:

Case 1: PS, optimized for speed: cell dimensions: $0.15 \mathrm{~mm} \times 0.15 \mathrm{~mm}$, 44000 cells, CPU Intel i3 $2.2 \mathrm{GHz}$ (3 MB cache)

Case 2: PS, optimized for RAM: cell dimensions: $0.15 \mathrm{~mm} \times 0.15 \mathrm{~mm}$, 44000 cells, CPU Intel i3 $2.2 \mathrm{GHz}$ (3 MB cache)

Case 3: ABAQUS, mesh $0.50 \mathrm{~mm} \times 0.50 \mathrm{~mm}, 4000$ nodes, CPU Intel i7 $2.2 \mathrm{GHz}$ (8 MB cache)

Case 4: $\quad$ ABAQUS, mesh $0.15 \mathrm{~mm} \times 0.15 \mathrm{~mm}, 44000$ nodes, CPU Intel i7 $2.2 \mathrm{GHz}$ (8 MB cache)

Dividing the simulated area into $\mathrm{n}$ areas, that are simultaneously simulated (depending on capabilities of the platform), divides the simulation time with the same factor $\mathrm{n}$ (some $10 \%$ added for synchronization of threads). It is realistic to simulate 10 seconds of simulated time in 1 second of real time on a budget platform. With this speed the simulation can become part of a real time control system. What is the technical cost for this fast simulation? Optimal dimensions of cells and length of $\Delta t$ have to be selected and verified in the simulation of the reference case. Adding the obvious: bigger cells and longer $\Delta t$ introduce larger errors for mathematical reasons, smaller cells and shorter $\Delta t$ introduce larger errors for rounding reasons and prolong the simulation time.

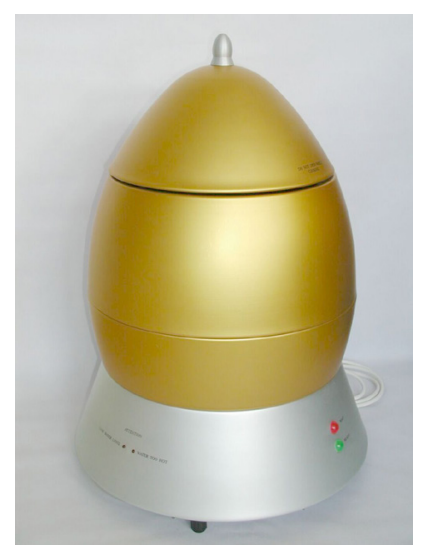

Fig. 10. Golden egg - producer of pasteurized soft boiled eggs
The derived thermal process is implemented in the apparatus in Fig. 10. The Golden egg produces pasteurized soft boiled eggs in batches of up to 30 eggs in 15 minutes intervals. Based on major industrial food preparation exhibitions, it is currently the only apparatus of its sort. The thermal process is patented.

\section{CONCLUSION}

A faster than real-time simulator of conductive heat transfer in a cylinder with a fixed simulation grid is developed. The presented simulation framework can be used for simulations of conductive heat transfer in different technical fields. It can be adapted to any problem where the local temporal intensity of changes depends on gradients and the properties of the matter. The case study is a simulation of heating-up an egg in hot water. The results of the study are used in the creation of a thermal process that is implemented in the Golden Egg apparatus which produces certified pasteurized soft boiled eggs.

\section{ACKNOWLEDGEMENT}

It was a pleasure to work with engineers of the $R \& D$ department of the Kogast d.d. when designing the GoldenEgg apparatus.

\section{REFERENCES}

[1] Barbour, E.K., El Jurdia, L., Issab, C., Tannousb, R. (2001). Preliminary attempts towards production of table eggs free from Salmonella enteritidis. Journal of Cleaner Production, vol. 9, no. 1, p. 69-73, Dol:10.1016/S0959-6526(00)00033-0.

[2] Hou, H., Singh, R.K., Muriana, P.M., Stadelman, W.J. (1996). Pasteurization of intact shell eggs. Food Microbiology, vol. 13, no. 2, p. 93-101, D0l:10.1006/fmic.1996.0012.

[3] Manas, P., Pagán, R., Alvarez, I., Condón Usón, S. (2003). Survival of Salmonella senftenberg $775 \mathrm{~W}$ to current liquid whole egg pasteurization treatments. Food Microbiology, vol. 20, no. 5, p. 593-600, Dol:10.1016/S0740-0020(02)00088-6.

[4] Schuman, J.D., Sheldon, B.W., Vandepopuliere, J.M., Ball, Jr.H.R. (1997). Immersion heat treatments for inactivation of Salmonella enteritidis with intact eggs. Journal of Applied Microbiology, vol. 83, no. 4, p. 438-444, D0I:10.1046/j.13652672.1997.00253.x.

[5] James, C., Lechevalier, V., Ketteringham, L. (2002). Surface pasteurization of shell eggs. Journal of Food Engineering, vol. 53, no. 2, p. 193-197, DOI:10.1016/S0260-8774(01)00156-X.

[6] Dev, S.R.S., Raghavan, G.S.V., Gariepy, Y. (2008). Dielectric properties of egg components and microwave heating for inshell pasteurization of eggs. Journal of Food Engineering, vol. 86, no. 2, p. 207-214, D0l:10.1016/j.jfoodeng.2007.09.027.

[7] Gao, M., Tang, J., Villa-Rojas, R., Wang, Y., Wang, S. (2011). Pasteurization process development for controlling Salmonella 
in in-shell almonds using radio frequency energy. Journal of Food Engineering, vol. 104, no. 2, p. 299-306, D0l:10.1016/j. jfoodeng.2010.12.021.

[8] Monfort, S., Saldana, G., Condon, S., Raso, J. (2012). Inactivation of Salmonella spp. in liquid whole egg using pulsed electric fields, heat, and additives. Food Microbiology, vol. 30, no. 2, p. 393-399, D0l:10.1016/j.fm.2012.01.004.

[9] Verde, S.C., Tenreiro, R., Botelho, M.L. (2004). Sanitation of chicken eggs by ionizing radiation: HACCP and inactivation studies. Radiation Physics and Chemistry, vol. 71, no 1-2, p. 27-31, D0l:10.1016/j.radphyschem.2004.03.064.

[10] Silva, F.V.M., Gibbs, P.A. (2012). Thermal pasteurization requirements for the inactivation of Salmonella in foods. Food Research International, vol. 45, no. 2, p. 695-699, Dol:10.1016/j.foodres.2011.06.018.

[11] Gut, J.A.W., Pinto, J.M., Gabas, A.L., Telis-Romero, J. (2005). Continuous pasteurization of egg yolk: thermo physical properties and process simulation. Journal of Food Process Engineering, vol. 28, no. 2, p. 181-203, D0l:10.1111/j.17454530.2005.00416.x.

[12] Romanoff, A.L., Romanoff, A.J. (1949). The Avian Egg. John Wiley and Sons, Hoboken, p. 150-169.
[13] Denys, S., Pieters, J.G., Dewettinck, K. (2003). Combined CFD and experimental approach for determination of the surface heat transfer coefficient during thermal processing of eggs. Journal of Food Science, vol. 68, no. 3. p. 943-951, D0l:10.1111/j.1365-2621.2003.tb08269.x.

[14] Ramachandran, R., Malhotra, D., Anishaparvin, D, Anandharamakrishnan, C. (2011). Computational fluid dynamics simulation studies on pasteurization of egg in stationary and rotation modes. Innovative Food Science and Emerging Technologies, vol. 12, no. 1, p. 38-44, D0l:10.1016/j.ifset.2010.11.008.

[15] Ozcan, S.E., Andriessens, S., Berckmans, D. (2010). Computational study of the heat transfer of an avian egg in a tray. Poultry Science, vol. 89, no. 4, p. 776-784, D0I:10.3382/ ps.2009-00230.

[16] Denys, S., Pieters, J.G., Dewettinck, K. (2004). Computational fluid dynamics analysis of combined conductive and convective heat transfer in model eggs. Journal of Food Engineering, vol. 63, no. 3, p. 281-290, D0l:10.1016/J.jfoodeng.2003.06.002. 